\title{
Reliability of rehabilitative ultrasound imaging for measuring the gluteus maximus muscle at rest and during contraction
}

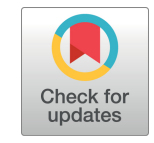

\author{
Ju-Ri Jeong ${ }^{a}$, Su-Jin Lee ${ }^{a}$, Wan-Hee Lee ${ }^{b}$ \\ ${ }^{a}$ Musculoskeletal Center, Seoul R\&D Campus Medical Clinic, Kangbuk Samsung Hospital, Seoul, Republic of Korea \\ ${ }^{b}$ Department of Physical Therapy, College of Health and Welfare, Sahmyook University, Seoul, Republic of Korea
}

\begin{abstract}
Objective: The aim of this study was to investigate the inter-rater and intra-rater reliability of rehabilitative ultrasound imaging (RUSI) for measurement of muscle thickness with changes in angles of the gluteus maximus (GM) at rest and during contraction. Design: Cross-sectional study.

Methods: Twenty-two healthy men volunteered for this study. GM muscle images were obtained in the resting position and during prone hip extension with knee flexion at hip abduction angles of $0^{\circ}$ and $30^{\circ}$, respectively. Two examiners randomly measured the thickness of the GM twice in three different positions. The first position was a comfortable prone position. The second position was prone hip extension with knee flexion (PHEKF) to $90^{\circ}$. The third position was achieved by hanging a 1-kg weight on the ankle of the lifted leg during PHEKF with the angle of the lifted leg the same as the second position. Intra-class correlation coefficients (ICCs), standard error measurements, and minimal detectable changes were used to estimate reliability.

Results: The intra-rater reliability ICCs (95\% confidence interval) of the GM were $>0.870$, indicating good reliability. Inter-rater reliability ICCs ranged from 0.668 to 0.913 . The reliability of measurements of muscle thickness at each position was similar to the reliability of the angle change. Differences in muscle thickness and ratios for each position with $0^{\circ}$ and $30^{\circ}$ of hip abduction were not statistically significant.

Conclusions: In the present study, the intra-rater reliability of muscle thickness measurements of the GM was good, and the inter-rater reliability was moderate to good. Reliable RUSI measurements of wide and large muscles, such as the GM muscle at rest and during contraction, are feasible. Further investigation is required to establish the reproducibility of the protocols presented in this study.
\end{abstract}

Key Words: Gluteus maximus, Muscle thickness, Reliability, Ultrasound

\section{Introduction}

Rehabilitative ultrasound imaging (RUSI) has been used advantageously by physiotherapists to measure muscle morphology. RUSI also can provide visual feedback as an exercise guide and can be used to measure both static and dynamic muscle tissue in the state of contraction [1-5]. RUSI has been used to address research and study questions regarding a variety of muscles [6]. Trunk, upper limbs, calf, and shin muscles have been the muscles most frequently researched using RUSI. However, except for one previous in- vestigation [6], studies utilizing RUSI to assess muscles of the buttocks are lacking.

The gluteal region is composed of the gluteus maximus (GM), gluteus medius, and gluteus minimus muscles. The GM is one of the largest and strongest muscles of the body $[1,7]$. In particular, the GM functions as a powerful extensor and abductor and is the, primary muscle in rehabilitation exercises for low back pain and lower-extremity injuries $[8,9]$. Furthermore, the GM plays a number of roles in the performance of activities such as walking, running, and lifting, and provides stability to the sacroiliac joint. The GM will affect

Received: 1 March, 2017 Revised: 14 March, 2017 Accepted: 16 March, 2017

Corresponding author: Wan-Hee Lee

Department of Physical Therapy, College of Health and Welfare, Sahmyook University, 815 Hwarang-ro, Nowon-gu, Seoul 01795, Republic of Korea Tel: 82-2-3399-1633 Fax: 82-2-3399-1639 E-mail: whlee@syu.ac.kr

(c) This is an Open-Access article distributed under the terms of the Creative Commons Attribution Non-Commercial License (http://creativecommons.org/licens es/by-nc/4.0) which permits unrestricted non-commercial use, distribution, and reproduction in any medium, provided the original work is properly cited.

Copyright $@ 2017$ Korean Academy of Physical Therapy Rehabilitation Science 
the kinetic chain change of the lower limb due to pain or injury [10]. For pelvic stability and safe and effective movement of the lower limbs, a GM strengthening exercise has been applied for rehabilitation training.

The GM strengthening exercise, prone hip extension with knee flexion (PHEKF) exercise, is a well-known, effective method for activation of the GM muscle during lower limb extension [11]. Recent research on application of the PHEKF exercise has used electromyography (EMG) and measurement of the angle of the leg to assess the activation of the GM [12]. Although many studies have evaluated GM activation using EMG, studies that measured GM activation using RUSI are lacking. An advantage of using RUSI to evaluate static and dynamic conditions of the muscle is reliable measurement of the GM state of change.

The purposes of this study were as follows: first, to establish a protocol to measure the GM muscle using RUSI; second, to determine whether measurement of the GM muscle with RUSI is highly reliable as it is with other muscles; and third, to investigate and compare the difference in muscle thickness as measured by the angle change between resting position and contraction of the GM muscle.

\section{Methods}

\section{Subjects}

Subjects were 22 young healthy (men recruited) from Sahmyook University students who volunteered to participate in this study. The participants had full, active, pain-free range of motion of prone hip extension with $90^{\circ} \mathrm{knee}$ flexion. Exclusion criteria were past or present musculoskeletal or neuromuscular disorders affecting the hip region and, history of hip pain in the preceding year. Obese individuals with body mass index (BMI) of $>30 \mathrm{~kg} / \mathrm{m}^{2}$ were also excluded [13]. The purpose of the study was explained to the participants, and informed consent was obtained. The study was approved by the Institutional Review Board of the Sahmyook University (IRB No. SYUIRB2015-17) in Seoul.

\section{Procedures}

An ultrasound imaging system (Medison Myosone U5; Samsung Medison Co. Ltd., Seoul, Korea) with a $12 \mathrm{MHz}$ linear transducer was used to obtain images of the GM muscle. Two examiners randomly measured the thickness of the GM twice in 3 different positions. The first position was a comfortable prone position (resting position). Each position comprised 2 kinds operations: first maintaining the legs side-by-side at $0^{\circ}$ on the prone table, then maintaining abduction at $30^{\circ}$. The second position was PHEKF with $90^{\circ}$ knee flexion. To achieve this, each participant lifted the dominant leg toward the ceiling until the patella was raised $5 \mathrm{~cm}$ off the table. The hip extension was then maintained for 5 seconds [14]. The leg was lifted at both $0^{\circ}$ and $30^{\circ}$ abduction as performed in the first position. The third position was achieved by hanging a 1-kg weight on the ankle of the lifted leg during PHEKF with the angle of the lifted leg the same as in the second position. Participants were instructed to rest and relax the hip and leg in the test positions. The process was repeated on the dominant side.

Prior to imaging, each participant's gluteal region was exposed in the prone position, and the dominant side was palpated and marked. To create exact RUSI scan protocols for the GM, we referred to previously published musculoskeletal ultrasound guidelines [15]. To determine the transducer placement, the examiners marked 2 lines from dominant side's posterior superior iliac spine to the ischial tuberosity
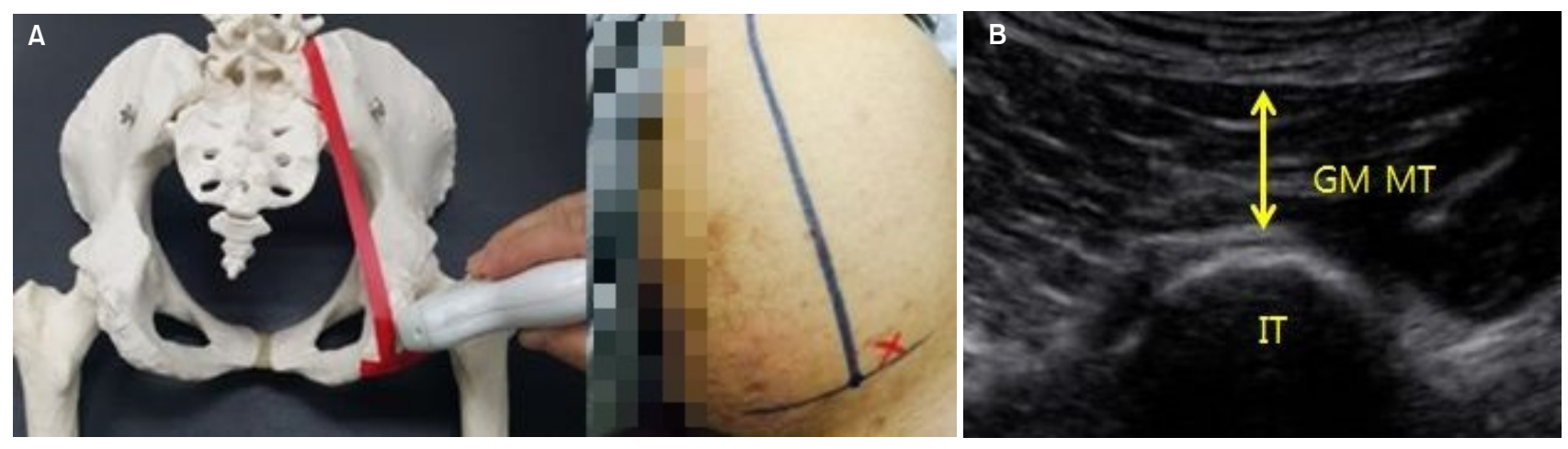

Figure 1. (A) Position of the probe to measure. (B) Rehabilitative ultrasound image of the GM. GM: gluteus maximus, MT: muscle thickness, IT: ischial tuberosity. 
(IT) and an imaginary line perpendicular from the previous line to the IT. The transducer was placed at the point where 2 lines intersected at a right angle (Figure 1A). The image was monitored to assure a continuous picture of the IT visualized at the middle part. The RUSI image showed skin, fat, GM, and IT (Figure 1B). The skin was mildly echogenic, and subcutaneous fat was hypoechoic on the RUSI scan. The GM is located between the IT and the hypo echoic area below the skin. The examiners measured the vertical length between the inferior echogenic fascial line of the skin and the superior hyperechoic region of the IT. In the study, to investigate the angle difference between the positions, the change in muscle thickness at $0^{\circ}$ to $30^{\circ}$ abduction was calculated, and the ratio of muscle thickness was determined.

\section{Data analysis}

Statistical analysis was performed using IBM SPSS
Statistics ver. 18.0 (IBM Co., Armonk, NY, USA). To describe the intra and inter-rater reliability of the GM muscle thickness at rest and during contraction, intra-class correlation coefficients (ICCs) were calculated [13]. Bland-Altman plots and $95 \%$ confidence intervals (CIs) were generated to provide a visual representation of muscle thickness to determine the agreement between the 2 measurements. ICC and Bland-Altman plots have been accepted as the statistical methods of choice in reliability studies. ICCs $<0.50$ were considered to represent poor, 0.50 to 0.75 moderate, and $>0.75$ high reliability [16]. To assess measurement accuracy, standard error of measurement (SEM) was calculated as standard deviation $\times \sqrt{1}-$ ICC. The $95 \%$ minimal detectable change $(\mathrm{MDC})$ was calculated as $1.96 \times \mathrm{SEM} \times \sqrt{2}$. One-way analysis of variance and independent t-test were used to compare the differences between and ratios of muscle thickness between positions between positions accord-

Table 1. Intra-rater between repeated measures for the gluteus maximus MT

$(\mathrm{N}=22)$

\begin{tabular}{|c|c|c|c|c|c|c|c|}
\hline & \multirow{2}{*}{ Variable } & \multicolumn{2}{|c|}{$\operatorname{MT}(\mathrm{cm})$} & \multirow{2}{*}{ ICC } & \multirow{2}{*}{$95 \% \mathrm{CI}$} & \multirow{2}{*}{ SEM } & \multirow{2}{*}{ MDC } \\
\hline & & $1 \mathrm{st}$ test & 2 nd test & & & & \\
\hline \multirow[t]{6}{*}{ E1 } & Resting $0^{\circ}$ & $2.32(0.58)$ & $2.41(0.62)$ & 0.938 & $0.850-0.974$ & 0.150 & 0.416 \\
\hline & Resting $30^{\circ}$ & $2.39(0.67)$ & $2.30(0.66)$ & 0.925 & 0.819-0.969 & 0.182 & 0.504 \\
\hline & Contraction $0^{\circ}$ & $3.22(0.54)$ & $3.24(0.57)$ & 0.967 & $0.921-0.986$ & 0.101 & 0.279 \\
\hline & Contraction $30^{\circ}$ & $3.20(0.62)$ & $3.29(0.71)$ & 0.947 & $0.872-0.978$ & 0.154 & 0.149 \\
\hline & Weight $0^{\circ}$ & $3.19(0.64)$ & $3.18(0.69)$ & 0.957 & $0.896-0.982$ & 0.149 & 0.413 \\
\hline & Weight $30^{\circ}$ & $3.32(0.71)$ & $3.32(0.73)$ & 0.892 & $0.739-0.955$ & 0.254 & 0.705 \\
\hline \multirow[t]{6}{*}{ E2 } & Resting $0^{\circ}$ & $2.40(0.82)$ & $2.37(0.86)$ & 0.954 & $0.888-0.981$ & 0.179 & 0.497 \\
\hline & Resting $30^{\circ}$ & $2.45(0.79)$ & $2.40(0.85)$ & 0.960 & $0.904-0.983$ & 0.163 & 0.452 \\
\hline & Contraction $0^{\circ}$ & $2.80(0.81)$ & $2.95(0.63)$ & 0.872 & $0.691-0.947$ & 0.259 & 0.718 \\
\hline & Contraction $30^{\circ}$ & $2.99(0.65)$ & $3.06(0.62)$ & 0.984 & $0.962-0.993$ & 0.081 & 0.224 \\
\hline & Weight $0^{\circ}$ & $3.00(0.71)$ & $2.85(0.71)$ & 0.903 & $0.766-0.960$ & 0.221 & 0.612 \\
\hline & Weight $30^{\circ}$ & $3.05(0.65)$ & $3.01(0.70)$ & 0.951 & $0.882-0.980$ & 0.150 & 0.415 \\
\hline
\end{tabular}

Values are presented as mean (SD).

MT: muscle thickness, ICC: intra-class correlation coefficient, CI: confidence interval, SEM: standard error of the measurement, MDC: minimal detectable change, E1: examiner first, E2: examiner second.

Table 2. Inter-rater reliability for gluteus maximus MT of repeated measure

$(\mathrm{N}=22)$

\begin{tabular}{|c|c|c|c|c|c|c|c|c|}
\hline \multirow{2}{*}{ Variable } & \multicolumn{4}{|c|}{1 st test } & \multicolumn{4}{|c|}{ 2nd test } \\
\hline & ICC & $95 \% \mathrm{CI}$ & SEM & $\mathrm{MDC}$ & ICC & $95 \% \mathrm{CI}$ & SEM & MDC \\
\hline Rest $0^{\circ}$ & 0.834 & $0.600-0.931$ & 0.439 & 1.217 & 0.832 & $0.595-0.930$ & 0.423 & 1.172 \\
\hline Resting $30^{\circ}$ & 0.691 & $0.256-0.872$ & 0.607 & 1.684 & 0.764 & $0.430-0.902$ & 0.440 & 1.220 \\
\hline Contraction $0^{\circ}$ & 0.668 & $0.201-0.862$ & 0.536 & 1.486 & 0.686 & $0.244-0.870$ & 0.512 & 1.420 \\
\hline Contraction $30^{\circ}$ & 0.788 & $0.489-0.912$ & 0.456 & 1.265 & 0.721 & $0.327-0.884$ & 0.485 & 1.343 \\
\hline Weight $0^{\circ}$ & 0.864 & $0.673-0.944$ & 0.385 & 1.066 & 0.833 & $0.597-0.930$ & 0.385 & 1.068 \\
\hline Weight $30^{\circ}$ & 0.755 & $0.409-0.898$ & 0.494 & 1.370 & 0.913 & $0.791-0.964$ & 0.277 & 0.767 \\
\hline
\end{tabular}

MT: muscle thickness, ICC: intra-class correlation coefficient, CI: confidence interval, SEM: standard error of the measurement, MDC: minimal detectable change. 


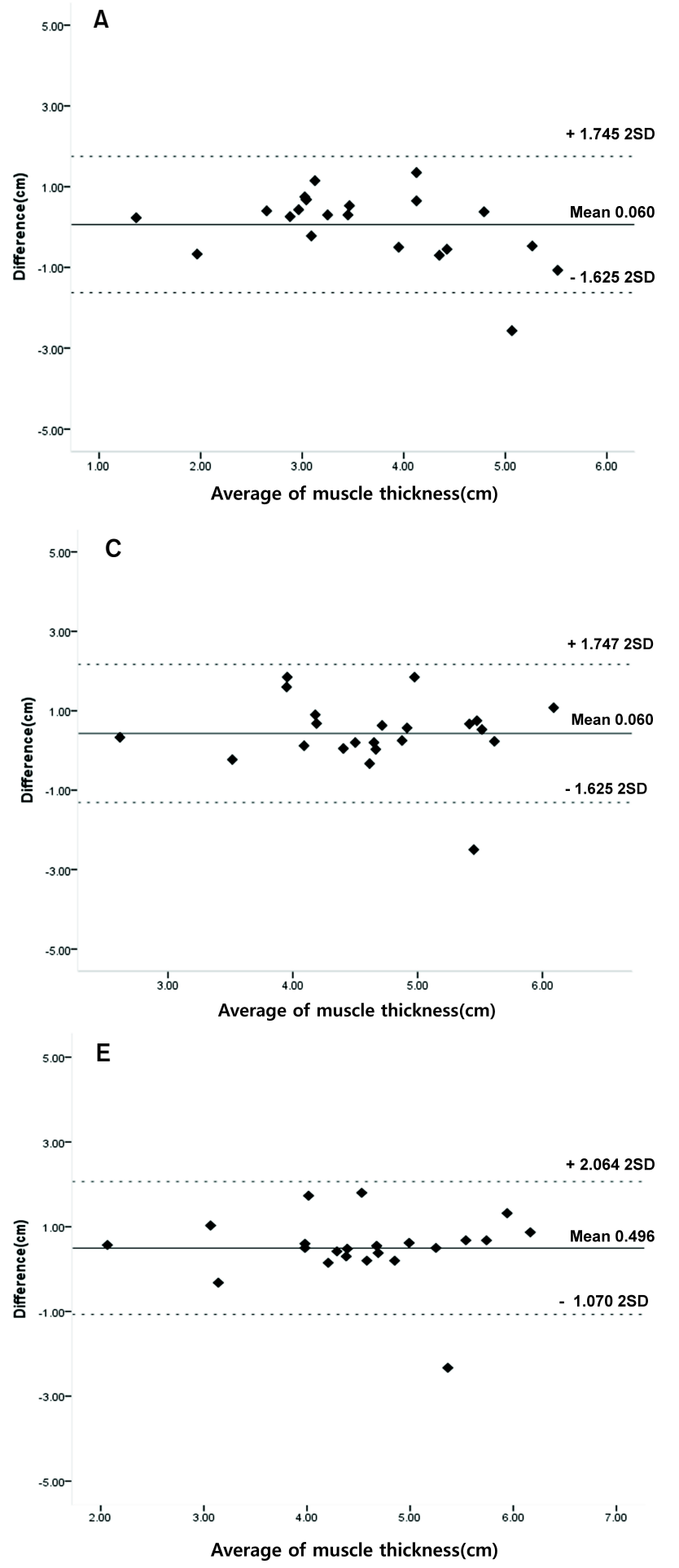

$5.00-\quad B$

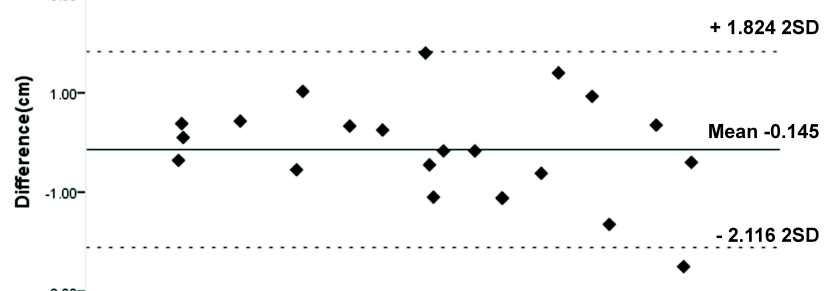

$-3.00-$

$-5.00^{-}$

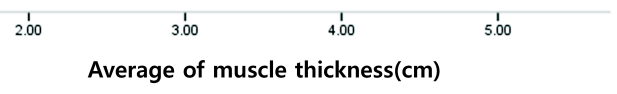

D

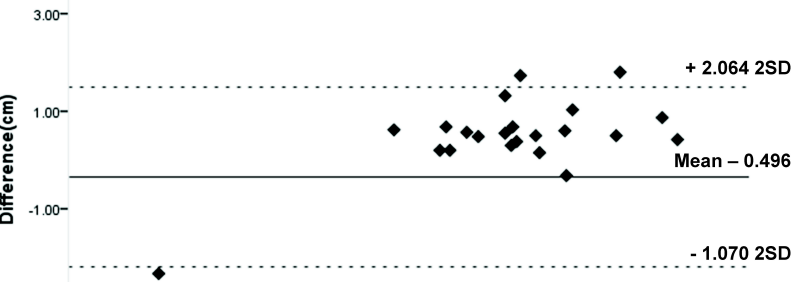

$-3.00-$

$.5 .00^{-}$

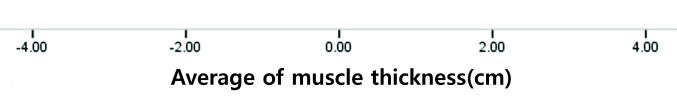

5.00- $\quad F$
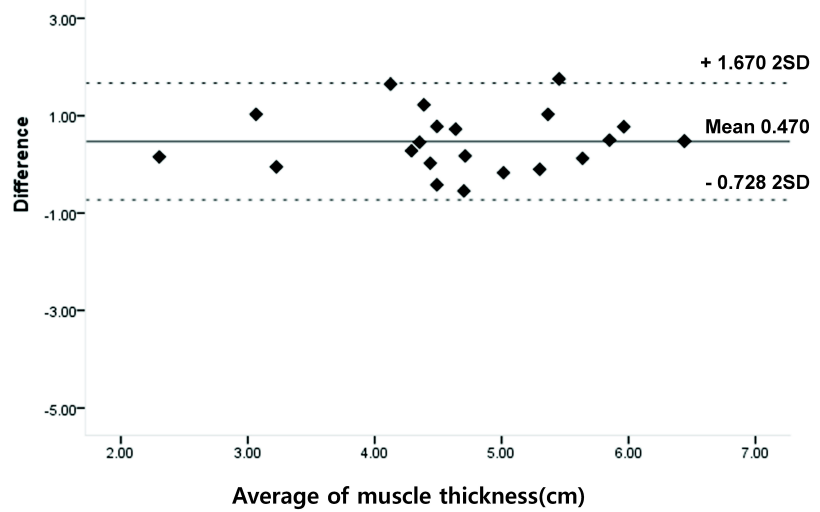

Figure 2. Bland-Altman plots illustrating the difference of rehabilitative ultrasound image for the gluteus maximus muscle between examiner 1 and 2 during the rest and contraction, weight contraction position for interrater reliability. (A) Resting position $0^{\circ}$ measurement. (B) Resting position $30^{\circ}$ measurement. (C) Contraction position $0^{\circ}$ measurement. (D) Contraction position $30^{\circ}$ measurement. (E) Weight contraction position $0^{\circ}$ measurement. (F) Weight contraction position $30^{\circ}$ measurement. SD: standard deviation. 
ing to angle changes. Statistical significance was set at $p<0.05$.

\section{Results}

\section{Demographic characteristics}

Characteristics of the participants were as follows: 22 healthy male participants with mean age $23.90 \pm 2.48$ years, mean weight $64.72 \pm 9.01 \mathrm{~kg}$, and mean height $172.04 \pm 5.71$ $\mathrm{cm}$. The mean BMI and thigh circumference were 21.79 \pm $2.34 \mathrm{~kg} / \mathrm{m}^{2}$ and $55.35 \pm 5.06 \mathrm{~cm}$, respectively.

\section{Intra-rater reliability analysis}

A summary of the results for the intra-examiner reliability of the GM muscle thickness and positions for the 2 sessions performed by 2 examiners is shown in Table 1 . The ICCs for intra-rater reliability ranged from 0.872 to 0.984 and the CI was within an acceptable range of 0.739 to 0.986 . The SEM values ranged from 0.081 to $0.259 \mathrm{~cm}$, and the MDC values ranged from 0.149 to 0.718 for the GM.

\section{Inter-rater reliability analysis}

The ICCs for inter-rater reliability ranged from 0.668 to 0.913 (Table 2). The SEM values ranged from 0.277 to 0.607 $\mathrm{cm}$, and the MDC values ranged from 0.767 to 1.684 . Bland-Altman plots for inter-rater reliability between examiners are shown in Figure 2.

\section{Ratio and difference of muscle thickness during positions}

No statistically significant differences were observed between $0^{\circ}$ and $30^{\circ}$ angle change positions (Table 3 ). No significant difference was seen in muscle thickness ratios for the 2 positions. However, the muscle thickness difference between $0^{\circ}$ and $30^{\circ}$ tended to increase in the more contracted state of $30^{\circ}$ abduction.

Table 3. The ratio and difference of muscle thickness on the gluteus maximus muscle according to the angle change $(\mathrm{N}=22)$

\begin{tabular}{cccc}
\hline \multicolumn{1}{c}{$\mathrm{MT}(\mathrm{cm})$} & Ratio & Difference & $p$ \\
\hline Position & & & \\
Resting $0^{\circ}-30^{\circ}$ & $1.03(0.17)$ & $0.04(0.42)$ & 0.726 \\
Contraction $0^{\circ}-30^{\circ}$ & $1.08(0.22)$ & $0.15(0.35)$ & 0.081 \\
Weight $0^{\circ}-30^{\circ}$ & $1.04(0.08)$ & $0.11(0.23)$ & 0.068 \\
$p$ & 0.551 & 0.594 & \\
\hline
\end{tabular}

Values are presented as mean (SD).

MT: muscle thickness.

\section{Discussion}

The GM muscle makes a large contribution to gait, effective lower limb movement, and pelvic stability [10]. In our study, the GM muscle was measured using RUSI. RUSI is a non-invasive, accessible, safe, and low-cost tool that is particularly effective for muscle size measurement [1]. Taking into account the direction (inferolateral) and point of attachment of the GM muscle fibers [7,17], we measured its thickness at angles of $0^{\circ}$ and $30^{\circ}$ abduction for each position. Furthermore, we modified existing musculoskeletal ultrasound guidelines [15] to develop a GM measurement protocol. Among the body's muscles, the GM is the largest, and its contraction results in a large volume change. Therefore, to accurately represent the landmark muscle, it was subjected to measurement in the same way for all participants.

In a previous study, in which the gluteus medius and minimus muscles were measured, intra-rater ICC achieved high reliability (all ICCs $>0.83$ ) [6]. In our study, RUSI of GM muscle thickness produced a good intra-rater reliability (E1 ICCs, 0.892 to 0.967 ; E2 ICCs, 0.872 to 0.960 ), but interrater reliability was moderate to good ( 1 st ICCs, 0.668 to 0.864 ; 2nd ICCs, 0.686 to 0.913 ). All 3 experimental conditions (resting, PHEKF, and PHEKF+1-kg weight) exhibited similar 95\% CI and ICCs. Inter-rater reliability was lower than intra-rater reliability. Reliability indices were similar for intra-rater, inter-rater, same-day, and separateday measurement of muscle thickness with RUSI. By contrast, in English et al.'s study [18], the reliability of posterior lower leg muscle thickness was moderate (intra-rater reliability $=0.70-0.71$ ), and in Talbott and Witt's study of the reliability of serratus anterior muscle thickness, inter-rater reliability at rest and during contraction was poor (ICCs, 0.425 to 0.526 ). Compared with the reliability of thickness of the abdominal muscles, which contract under relatively static conditions $[19,20]$, the reliability of thickness of muscles that contract under dynamic conditions is lower [13]. SEMs of intra-rater reliability for both the first and second examiner were similar (E1 SEM, 0.101 to $0.254 \mathrm{~cm}$; E2 $\mathrm{SEM}, 0.081$ to $0.259 \mathrm{~cm}$ ), whereas SEM values of inter-rater reliability (SEM, 0.385 to $0.607 \mathrm{~cm}$ ) were higher.

Muscle thickness was found to be greater at $30^{\circ}$ than at $0^{\circ}$ hip abduction, but the difference was not statistically significant. Similarly, no significant difference was observed even when muscle thickness was compared with the ratio increase. However, in the weight contraction, muscle thick- 
ness tended to be thicker. This study was designed to assess results at angles of $0^{\circ}$ and $30^{\circ}$ hip abduction in accordance with the findings of Kang et al. [12] study. In Kang et al.'s study of muscle contraction with hip abduction of $0^{\circ}, 15^{\circ}$, and $30^{\circ}$ during PHEKF exercise, GM muscle activation at $30^{\circ}$ abduction increased significantly $(p<0.001)$. However, the EMG measurement point in that study was in the upper fibers of the GM, whereas in our study, the ultrasound measurement point was in the lower fibers. The GM's lower fibers play a greater role in hip abduction extensor force compared with that of the upper fibers [21]. Changes in the muscle in regard to angle of abduction in the present study did not significantly, increase muscle thickness beyond that expected as a result of the contracting force of hip extension. In consideration of the fiber arrangement of the GM, it was necessary to create a RUSI protocol that used measurements of the upper fibers. In addition, the difference in muscle thickness due to different angles of abduction affected the sample size and significance of results.

This study had several limitations. First, all participants were young males. Second, the sample size was small. Third, the accuracy and reproducibility of the results need to be confirmed because the experimental protocol of the study has never been used before. Fourth, because the GM is a large and bulky muscle, it is necessary to control the conditions under which its contraction can be measured.

This study demonstrates that GM muscle thickness can be measured reliably using RUSI. However, as in measurement of other muscles, intra-rater reliability was higher than the inter-rater reliability in measurement of GM muscle thickness with RUSI. Further experimentation is needed to increase the reproducibility of RUSI results. Nevertheless, in patients with limping gait or severe muscle atrophy, measurement of GM thickness may be useful. Thus, application of the research may widen the practical use of RUSI.

\section{Acknowledgements}

The authors thank the students of the Sahmyook University for participating in this study.

\section{Conflict of Interest}

The authors declared no potential conflicts of interest with respect to the authorship and/or publication of this article.

\section{References}

1. Whittaker JL, Teyhen DS, Elliott JM, Cook K, Langevin HM, Dahl HH, et al. Rehabilitative ultrasound imaging: understanding the technology and its applications. J Orthop Sports Phys Ther 2007;37:434-49.

2. Ko YJ, Ha HG, Jeong J, Lee WH. Variations in lateral abdominal muscle thickness during abdominal drawing-in maneuver in three positions in a young healthy population. Phys Ther Rehabil Sci 2014;3:101-6.

3. Yoo JS, Ha HG, Jeong JR, Ko YJ, Lee W. Physical therapist perception survey for muscle re-education through visual feedback obtained from rehabilitative ultrasound imaging. Phys Ther Rehabil Sci 2016;5:47-52.

4. Jeong JR, Han JH, Cho JE, Lee W. Reliability and validity of a personal computer based muscle viewer for measuring upper trapezius and transverses abdominis muscle thickness. Phys Ther Rehabil Sci 2016;5:155-61.

5. Kim TH, Hahn J, Jeong JR, Lee C, Kim YJ, Choi SM, et al. Changes of abdominal muscle thickness during stable and unstable surface bridging exercise in young people. Phys Ther Rehabil Sci 2016;5:210-4.

6. Whittaker JL, Emery CA. Sonographic measures of the gluteus medius, gluteus minimus, and vastus medialis muscles. J Orthop Sports Phys Ther 2014;44:627-32.

7. Netter FH. The ciba collection of medical illustrations: vol 8: musculoskeletal system: part I: anatomy, physiology and metabolic disorders. Lincoln: Anybook Ltd; 1987.

8. Lehman GJ, Lennon D, Tresidder B, Rayfield B, Poschar M. Muscle recruitment patterns during the prone leg extension. BMC Musculoskelet Disord 2004;5:3.

9. Day JM, Uhl T. Thickness of the lower trapezius and serratus anterior using ultrasound imaging during a repeated arm lifting task. Man Ther 2013;18:588-93.

10. Wilson J, Ferris E, Heckler A, Maitland L, Taylor C. A structured review of the role of gluteus maximus in rehabilitation. $\mathrm{J}$ Physiother 2005;33:95-100.

11. Sakamoto ACL, Teixeira-Salmela LF, Rodrigues de Paula F, Guimarães CQ, Faria CDCM. Gluteus maximus and semitendinosus activation during active prone hip extension exercises. Bras J Phys Ther 2009;13:335-42.

12. Kang SY, Jeon HS, Kwon O, Cynn HS, Choi B. Activation of the gluteus maximus and hamstring muscles during prone hip extension with knee flexion in three hip abduction positions. Man Ther 2013;18:303-7.

13. Talbott NR, Witt DW. Ultrasound imaging of the serratus anterior muscle at rest and during contraction. Clin Physiol Funct Imaging 2013;33:192-200.

14. Dankaerts W, O'Sullivan PB, Burnett AF, Straker LM, Danneels LA. Reliability of EMG measurements for trunk muscles during maximal and sub-maximal voluntary isometric contractions in healthy controls and CLBP patients. J Electromyogr Kinesiol 2004; 14:333-42.

15. Martinoli C. Musculoskeletal ultrasound: technical guidelines. Insights Imaging 2010;1:99-141.

16. Portney LG, Watkins MP. Foundations of clinical research: ap- 
plications to practice. 2nd ed. Upper Saddle River, NJ: Prentice Hall Health; 2000.

17. McAndrew D, Gorelick M, Brown JMM. Muscles within muscles: a mechanomyographic analysis of muscle segment contractle properties within human gluteus maximus. J Musculoskelet Res 2006;10:23-35.

18. English C, Fisher L, Thoirs K. Reliability of real-time ultrasound for measuring skeletal muscle size in human limbs in vivo: a systematic review. Clin Rehabil 2012;26:934-44.

19. Koppenhaver SL, Hebert JJ, Fritz JM, Parent EC, Teyhen DS,
Magel JS. Reliability of rehabilitative ultrasound imaging of the transversus abdominis and lumbar multifidus muscles. Arch Phys Med Rehabil 2009;90:87-94.

20. Gnat R, Saulicz E, Miądowicz B. Reliability of real-time ultrasound measurement of transversus abdominis thickness in healthy trained subjects. Eur Spine J 2012;21:1508-15.

21. Barker PJ, Hapuarachchi KS, Ross JA, Sambaiew E, Ranger TA, Briggs CA. Anatomy and biomechanics of gluteus maximus and the thoracolumbar fascia at the sacroiliac joint. Clin Anat 2014; 27:234-40 